\title{
Modeling and numerical simulation of the nonlinear dynamics of the parametrically forced string pendulum
}

\author{
Veronica Ciocanel* \\ Advisor: Thomas Witelski ${ }^{\dagger}$
}

June 12, 2012

\begin{abstract}
The string pendulum consists of a mass attached to the end of an inextensible string which is fastened to a support. Applying an external forcing to the pendulum's support is motivated by understanding the behavior of suspension bridges or of tethered structures during earthquakes. The forced string pendulum can go from taut to slack states and vice versa, and is capable of exhibiting interesting periodic and chaotic dynamics. The inextensibility of the string and its capacity to go slack make simulation and analysis of the system complicated. The string pendulum system is thus formulated here as a piecewise-smooth dynamical system using the method of Lagrange multipliers to obtain a system of differential algebraic equations (DAE) for the taut state.

In order to develop a formulation for the forced string pendulum system, we first turn to similar but simpler pendulum systems, such as the classic rigid pendulum, the elastic spring pendulum and the elastic spring pendulum with piecewise constant stiffness. We perform a perturbation analysis for both the unforced and forced cases of the spring pendulum approximation, which shows that, for large stiffness, this is a reasonable model of the system. We also show that the spring pendulum with piecewise constant stiffness can be a good approximation of the string pendulum, in the limit of a large extension constant and a low compression constant. We indicate the behavior and stability of this simplified model by using numerical computations of the system's Lyapunov exponents. We then provide a comparison of the spring pendulum with piecewise constant stiffness with the formulation of the taut-slack pendulum using the DAE for the taut states and derived switching conditions to the slack state.
\end{abstract}

\section{Introduction}

The string pendulum, which consists of a mass suspended by a string, is a seemingly simple system displaying interesting behavior under the application of various external

${ }^{*}$ Duke University, Department of Mathematics; e-mail: veronica.ciocanel@duke.edu

${ }^{\dagger}$ Professor, Duke University, Department of Mathematics; e-mail: witelski@math.duke.edu 


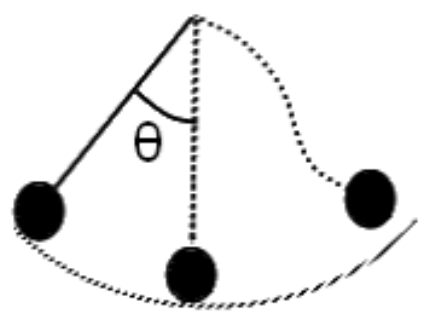

(a)

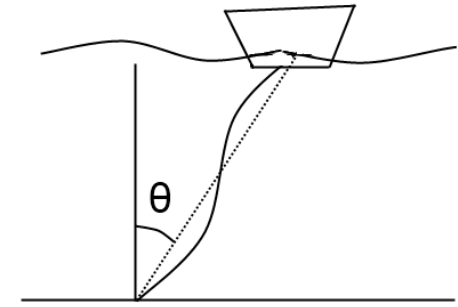

(b)

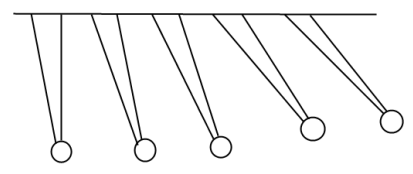

(c)

Figure 1: A string pendulum (a) and its applications such as (b) moored boats and (c) Newton's cradle

forces. Modeling this system can become challenging if we consider that the string can become slack and investigate its stability behavior. The forced string pendulum problem introduces changes of states that determine the non-smooth character of the system, thus leading to ODE's with switching forms, which are piecewise smooth [11]. Kinematic constraints or physical effects such as friction, impacts or backlash are known to cause non-smooth phenomena which represent a challenge for engineers and mathematicians [11]. Moreover, since these problems are nonlinear, chaotic motions can appear.

The classic rigid pendulum has been studied extensively as a model of many simple nonlinear oscillators. The planar classic pendulum with vertical periodic forcing was studied in [1], and its sensitivity to initial conditions was inspected by looking at the system's Lyapunov exponents. In [5], it was shown that for large-enough velocities, the string pendulum will become slack and the mass will follow a parabolic path for projectile motion. Taut-slack states can occur in this situation, however no external forcing is considered. In [7], the motion of a jogger's ponytail is modeled using first a rigid string and then a flexible string under periodic vertical forcing, but the stability of the system is not discussed. An experimental approach to analyze the behavior of two cables attached to a rigid frame and shaken horizontally was proposed in [1].

We consider basic mechanical systems of pendulums, as well as use the physical understanding of the system to guide us to mathematical approximations of the string forced pendulum. This work is motivated by broader applications to ODE's with switching forms and also to specific extensions of the forced string pendulum.

One application is Newton's cradle, which is a desktop toy that shows the effects of conservation of energy and momentum, as well as those of friction and air resistance. It usually consists of 5 metal balls which are touching at rest, each suspended by strings from a common rigid frame, see Fig. 1c. This toy is usually observed for its reaction to the action of pulling one or two of its exterior balls and letting them collide. However, more interesting dynamics involving impacts and chaotic motion can be noticed when pulling one or two balls sideways so that one of the strings is taut and one slack [15]. Moreover, the behavior of the system when the whole frame is moved either horizontally or vertically with a given forcing can be of interest. Similarly, analyzing the dynamics 


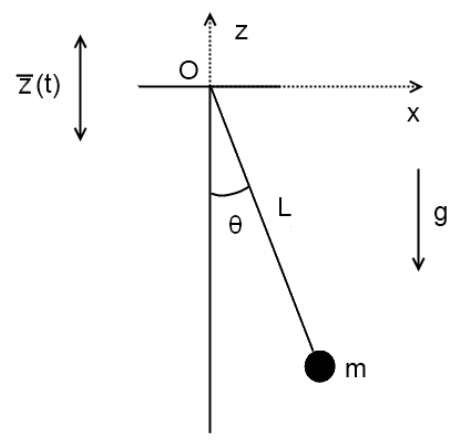

Figure 2: A schematic diagram of the rigid pendulum with vertical motion $\bar{z}(t)$ of the pivot

of structures during earthquakes could benefit from a study of forced frames.

Another application is describing moored boats, which are fastened to a fixed foundation by a rope, see Fig. 1b. Boats anchored during storms could undergo backlash and become loose if the rope is fully extended, thus becoming analogous to the model of a pendulum with loose string. Another example of a system with switching behavior is that of suspension bridges, whose loads are hung on suspension cables. These cables are normally linear at equilibrium, but if shaking occurs they can slacken, leading to piecewise defined characteristics [6].

Our main goal is to explain and analyze such interesting dynamics. However, to move up to this goal, we need to first analyze the mathematics of simpler systems. We start by analyzing the dynamics of the classic pendulum with forcing in Section 1.1. Section 2 is dedicated to the modeling of the classic and taut-slack pendulum using a spring pendulum. Section 3 shows the modeling of the classic pendulum system using a DAE system to impose the geometric constraint of fixed length. The changes that need to be considered when switching to the taut-slack pendulum system are analyzed in Section 4. Lastly, Section 5 provides a comparison of the DAE and spring pendulum models of the taut-slack case.

\subsection{Reference model: The classic pendulum}

We first review the equations of motion of a classic pendulum (with rigid, non-stretchable string) attached to a support subjected to a prescribed vertical motion $\bar{z}(t)$ of the pivot point, see Fig. 2.

The geometric description for accessible positions of the pendulum mass is

$$
x(t)=L \sin \theta(t), \quad z(t)=\bar{z}(t)-L \cos \theta(t),
$$

where $L$ is the length of the pendulum and the reference frame is stationary.

The kinetic and gravitational potential energy of the system are defined as

$$
T=\frac{1}{2} m\left(\dot{x}^{2}+\dot{z}^{2}\right), \quad U=m g z,
$$

where $m$ is the mass of the pendulum. 

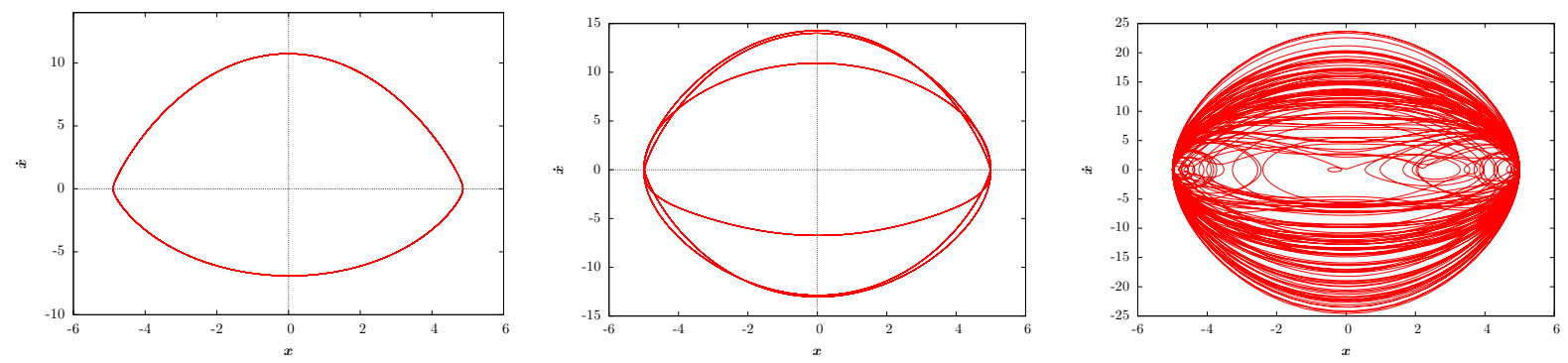

Figure 3: Phase planes of $x$ vs. $\dot{x}$ for the forced damped pendulum for amplitude $A=4 \mathrm{~m}$ (simple periodic), $A=8.35 \mathrm{~m}$ (higher order periodic oscillations), and $A=11 \mathrm{~m}$ (chaotic motion) using equation (1.4). Parameter values used are $L=5 \mathrm{~m}, g=9.8 \mathrm{~m} / \mathrm{s}, \omega=$ $0.9 \sqrt{g / L} \mathrm{~s}^{-1}$ and damping $\beta=0.01 \mathrm{~kg} / \mathrm{s}$.

We will use Hamilton's principle to formulate the governing equations for the system [8], in terms of the Euler-Lagrange equations [10]:

$$
\frac{\partial \mathscr{L}}{\partial u}-\frac{d}{d t}\left(\frac{\partial \mathscr{L}}{\partial \dot{u}}\right)=\frac{\partial F}{\partial \dot{u}}
$$

where $u(t)$ represents the state of the system (in this case $u=\theta(t)$ ) and $F$ is a Rayleigh dissipation function. This function describes damping terms in the equation of motion, which account for friction and other non-conservative effects that might exist in the system.

In our case, the Lagrangian takes the form

$$
\mathscr{L}=T-U=\frac{1}{2} m\left(L^{2} \dot{\theta}^{2}+\dot{\bar{z}}^{2}+2 L \dot{\theta} \dot{\bar{z}} \sin \theta\right)-m g \bar{z}+m g L \cos \theta,
$$

and the dissipation function is $F=\frac{\beta}{2}|\boldsymbol{v}|^{2}$, where $\boldsymbol{v}$ is the velocity and $|\boldsymbol{v}|^{2}=\dot{x}^{2}+\dot{z}^{2}$.

Now we can proceed to finding $\frac{\partial \mathscr{L}}{\partial \theta}$ and $\frac{\partial \mathscr{L}}{\partial \dot{\theta}}$ and plugging them in (1.2) yields the equation of motion for $\theta$ :

$$
\ddot{\theta}=-\frac{g+\ddot{\bar{z}}}{L} \sin \theta-\frac{\beta}{m} \dot{\theta}-\frac{\beta}{m L} \dot{\bar{z}} \sin \theta .
$$

For the planar pendulum with no external forcing $(\bar{z}=0)$ and no damping $(\beta=0)$, the model reduces to the simple pendulum equation. The case of no damping and external forcing $\bar{z}$, together with linearization for small angles, is the linear Mathieu equation with parametric excitation of the system. This forced pendulum (1.4) will be used as a comparison to check the behaviors produced by the other models.

If we consider

$$
\bar{z}(t)=A \cos (\omega t),
$$

and small damping $\beta$, then we can analyze the stability of the solution of equation (1.4). Different amplitudes $A$ of the vertical forcing will produce qualitatively different solutions. The equations are solved numerically using the fourth order Runge-Kutta method [12]. Fig. 3a shows the phase plane for a stable periodic solution and Fig. 3c 


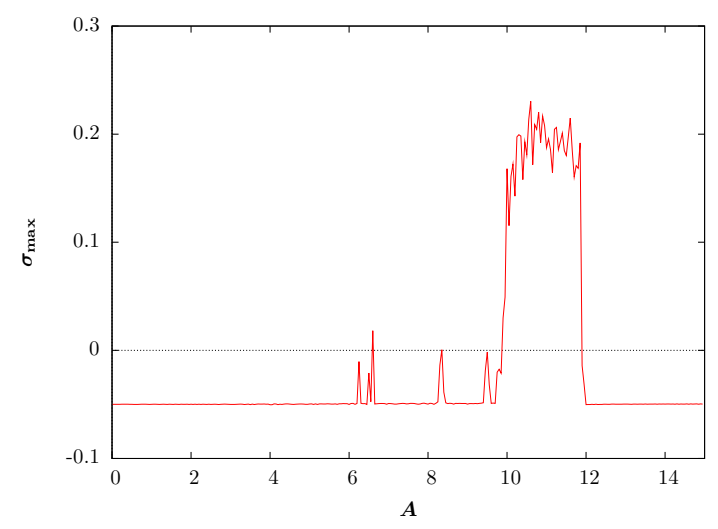

Figure 4: Parameter dependence of the largest Lyapunov exponent for a range of forcing amplitudes for the forced damped pendulum. Parameter values used are $L=5.0, g=9.8$, $\omega=0.9 \sqrt{g / L}, \beta=0.1$.

for a chaotic solution. Some values of the parameter $A$ (which correspond to spikes to 0 in the Lyapunov exponent plot in Fig. 4) result in more complicated oscillations in the phase planes which are part of a series of bifurcations between simple periodic and chaotic solutions. An example is given in Fig. 3b for $A=8.35$. These behaviors hold systematically for most initial conditions the system can be started on and seem to depend on the forcing parameter value. We thus vary the forcing amplitude $A$ and fix the other parameters to typical values, such as small damping $\beta$. A more thorough study could also take into account dependence on other parameter values.

Lyapunov exponents are a way of qualitatively and quantitatively characterizing systems' dynamical behavior. Lyapunov exponents determine a system's exponential divergence or convergence of nearby orbits in phase space. This is a way of showing sensitive dependence on initial conditions, meaning that neighboring orbits separate exponentially fast [14] and that solution behavior is thus unpredictable. Having one or more positive Lyapunov exponents defines a system as being chaotic [14], [16].

It is therefore important to identify the largest Lyapunov exponent of a system. In order to compute this, we consider a trajectory in phase space, $\boldsymbol{u}_{\mathbf{0}}(t)$, given by the nonlinear equations of the system applied to some initial condition. If we consider another trajectory starting nearby, $\boldsymbol{u}_{\mathbf{1}}(t)$, and set $\Delta \boldsymbol{u}=\boldsymbol{u}_{\mathbf{0}}-\boldsymbol{u}_{\mathbf{1}}$, then the logarithm of this difference represents the rate of exponential divergence or convergence: $\sigma=$ $\ln |\Delta \boldsymbol{u}| / \Delta t$. When the length of the vector between the reference trajectory and a trajectory starting nearby becomes large, we choose a new trajectory close to the reference trajectory and repeat the process on the next time interval.

The methods for determining the Lyapunov exponents outlined in [16] and [2] use linearized ODE's for $\boldsymbol{u}_{\mathbf{1}}$. These methods could be extended and applied to the system of DAE's (differential algebraic equations) to be later discussed in section 3 by using linearized DAE systems; however, this would require more work since the Wolf and Rugh methods were designed for ODE systems. In order to compute the exponential divergence or convergence of trajectories in such a situation, we write, for $\left[t_{n}, t_{n+1}\right]$ in a sequence of time intervals: $\Delta \boldsymbol{u}\left(t_{n+1}\right)=\Delta \boldsymbol{u}\left(t_{n}\right) e^{\sigma\left(t_{n+1}-t_{n}\right)}$. When $0.1<\frac{\left|\Delta \boldsymbol{u}\left(t_{n+1}\right)\right|}{\left|\Delta \boldsymbol{u}\left(t_{n}\right)\right|}<10$ 
ceases to be satisfied, which means that the length of the vector between the two points has become too large or too small, the next time interval is considered: $\left[t_{n}, t_{n+1}\right]$ is reset with $n \rightarrow n+1$. The largest Lyapunov exponent is thus given by the average growth rates for all of these time intervals [16]:

$$
\sigma_{\max }=\lim _{n \rightarrow \infty} \frac{1}{n} \sum_{n} \frac{1}{t_{n+1}-t_{n}} \ln \frac{\left|\Delta \boldsymbol{u}\left(t_{n+1}\right)\right|}{\left|\Delta \boldsymbol{u}\left(t_{n}\right)\right|} .
$$

In Fig. 4 we plot the largest Lyapunov exponent of the system versus the amplitude of the vertical forcing. We note how different values of $A$ correspond to a chaotic $(A=11)$ or periodic $(A=1)$ solution, and the phase planes in Fig. 3 correspond to intervals of stable $(A=4)$ and unstable $(A=11)$ amplitudes. The Lyapunov exponents will provide us with a way to compare different ways of modeling the pendulum systems considered. We propose the conjecture that comparable models will be similar in their stability behavior and thus have similar Lyapunov exponents, $\sigma_{\max }(A)$. This hypothesis is supported by the exponents plotted in Figs. 4, 6 and 9 .

\section{The spring pendulum}

The derivation of equation (1.4) started with the geometric constraint on the length of the pendulum imposed through equations (1.1). As we will see in Section 3, imposing the constraint of fixed length after writing the Euler-Lagrange equations creates more mathematical challenges. But such an approach will nevertheless allow for generalizing the problem, so it is of value for our later analysis. As a comparison problem, we first consider the dynamics for a spring pendulum, where the pendulum length can vary. Such a model is considered in [5], though no external force is applied to the system in that case.

The spring pendulum is a physical system with a mass connected to a spring so that the motion that results has characteristics of a simple pendulum as well as a spring. The spring is restricted to lie in a straight line, which can be achieved by wrapping the spring around a rigid massless rod [9]. Such a system is also called a general "elastic pendulum" in [13]. We will consider such a spring pendulum with a large Hooke's law spring constant $k$ which makes it moderately stiff, as well as a damping constant $\beta$ which helps us control the stretching oscillation of the spring. We consider a pendulum made up of a spring with mass $m$ on the end and let the angle it makes with the vertical be denoted by $\theta(t)$. The pendulum length $\tilde{L}=L-\frac{m g}{k}$ is selected so that the equilibrium length at $\theta=0$ is $L$ (see Fig. 5). The pendulum will thus be described as having effective length $L+r(t)$ at time $t$ where $r$ is the relative stretching of the spring.

As before, the spring pendulum will have an imposed vertical forcing which represents a motion of the pendulum pivot point, $\bar{z}$ acting on it vertically. The results obtained will be compared against the behavior determined in equation (1.4).

The Lagrangian is

$$
\mathscr{L}=T-U=\frac{1}{2} m\left(\dot{x}^{2}+\dot{z}^{2}\right)-\left[m g z+\frac{1}{2} k\left(r+\frac{m g}{k}\right)^{2}\right],
$$




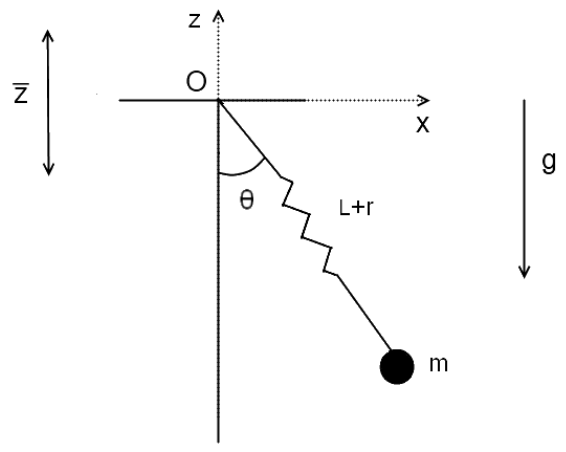

Figure 5: A schematic diagram of a spring pendulum with un-stretched length $\tilde{L}$ and stretch $r$, making an angle $\theta$ with the vertical

with $x$ and $z$ defined in this case as

$$
x=(L+r) \sin \theta, \quad z=\bar{z}-(L+r) \cos \theta .
$$

Now we can apply the Euler-Lagrange equations with respect to $\boldsymbol{u}=(r, \theta)$. We again consider a Rayleigh dissipation function of the form $F=\beta|\boldsymbol{v}|^{2} / 2$. The EulerLagrange equations are:

$$
\begin{gathered}
\ddot{r}=\ddot{\bar{z}} \cos \theta+(L+r) \dot{\theta}^{2}+g \cos \theta-\frac{k r}{m}-g-\frac{\beta}{m} \dot{r}+\frac{\beta \dot{\bar{z}}}{m} \cos \theta, \\
\ddot{\theta}=-\frac{g+\ddot{\bar{z}}}{L+r} \sin \theta-\frac{2}{L+r} \dot{r} \dot{\theta}-\frac{\beta}{m} \dot{\theta}-\frac{\beta \dot{\bar{z}}}{m(L+r)} \sin \theta .
\end{gathered}
$$

Equation (2.2) produces solutions that are very similar to the behavior given by equation (1.4). Time profiles of $\theta(t)$ for equation (2.2) match very well those from equation (1.4), and the small differences come from the small oscillations in the extension $r(t)$. Figure 6 shows a similar chaotic solution to the one in picture $3 \mathrm{c}$ ) for the same amplitude $A=11$.

In Fig. 6a we plot the largest Lyapunov exponent of the spring pendulum system versus the amplitude of the vertical forcing. We note that, for smaller values of $k$ such as $k=250$ or $k=500$, the Lyapunov exponent evolution is shifted to the right when compared to Fig. 4 and shows sharp drops, while for $k \rightarrow \infty$ the evolution matches Fig. 4 very well. The largest Lyapunov exponent of the classic pendulum is plotted on the same graph and shows that this system has some higher values of the Lyapunov exponent for certain amplitudes $A$ when compared to the spring pendulum. Moreover, the ranges of chaotic behavior are slightly larger for the classic pendulum. However, Fig. 6a shows an overall very good match between the Lyapunov exponents of the classic and spring pendulums, which verifies our expectation that the Lyapunov exponent evolution of the two modeling methods should be similar.

We can explore the comparison of the spring pendulum to the classic pendulum more carefully by doing a perturbation analysis of the spring pendulum system. We will 

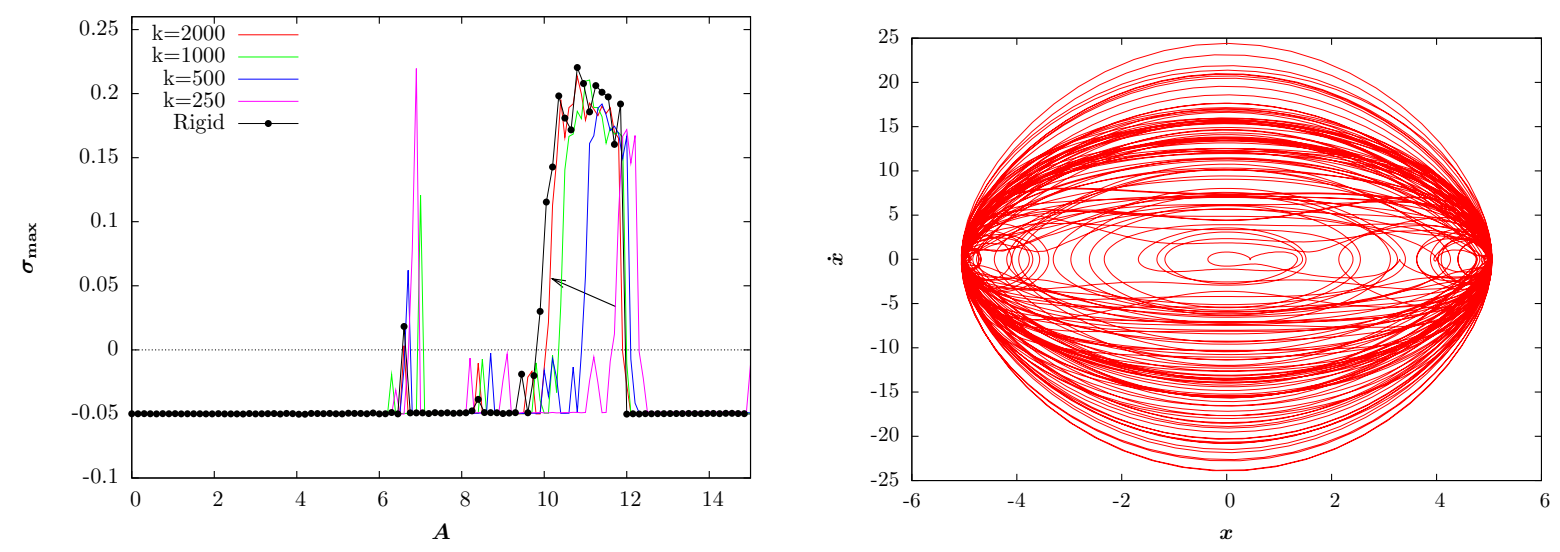

Figure 6: a) The progression of the largest Lyapunov exponent for amplitude $A$ ranging from 0 to 15 at different spring constants $k$. Parameter values used are $L=5, g=9.8$, $\omega=0.9 \sqrt{g / \tilde{L}}$ and $\beta=0.1$. b) Phase plane of $x$ vs. $\dot{x}$ for the spring pendulum for amplitude $A=11$.

consider the case of small amplitude oscillations and define our asymptotic parameter as the initial angular position, $\theta(0)=\epsilon \ll 1$. We write the expansions

$$
\theta \sim \epsilon \theta_{1}(t)+\epsilon^{2} \theta_{2}(t)+\ldots, \quad r \sim r_{0}(t)+\epsilon r_{1}(t)+\epsilon^{2} r_{2}(t)+\ldots,
$$

We use the above expansions in equation (2.2a) and since for $\epsilon \rightarrow 0, \cos \theta \sim 1-\frac{\epsilon^{2} \theta_{1}^{2}}{2}$, the $\mathscr{O}(1)$ terms yield the equation for $r_{0}$ :

$$
\ddot{r_{0}}+\frac{\beta}{m} \dot{r_{0}}+\frac{k}{m} r_{0}=\ddot{\bar{z}}+\frac{\beta}{m} \dot{\bar{z}} .
$$

Equation (2.4) describes a damped, non-resonant linear oscillator and can be solved for our choice of external forcing given in (1.5), with initial conditions $r(0)=0, \dot{r}(0)=$ 0 . This means that for large spring constant $k, r_{0}$ will converge to the particular solution from the $\bar{z}$ forcing.

Now we consider equations (2.2) in the limit of a very large spring constant $k=\frac{1}{\epsilon}$, $\epsilon \rightarrow 0$ and small length perturbations $r=\epsilon \tilde{r}$. In (2.2a), this yields:

$$
\epsilon \ddot{\tilde{r}}=\ddot{\bar{z}} \cos \theta+(L+\epsilon \tilde{r}) \dot{\theta}^{2}+g \cos \theta-g-\frac{\tilde{r}}{m}-\frac{\beta}{m} \epsilon \tilde{r}+\frac{\beta}{m} \dot{\bar{z}} \cos \theta .
$$

Using the expansion $\tilde{r} \sim \tilde{r}_{0}(t)+\epsilon \tilde{r}_{1}(t)+\epsilon^{2} \tilde{r}_{2}(t)+\ldots$, the $\mathscr{O}(1)$ terms give the following equation for $\tilde{r}_{0}$ :

$$
\tilde{r}_{0}=m g(\cos \theta-1)+m \ddot{\bar{z}} \cos \theta+m L \dot{\theta}^{2}+\beta \dot{\bar{z}} \cos \theta .
$$

If we assume that $\dot{\theta}$ is bounded (and thus $\dot{\theta}^{2}$ is bounded), then the equation for $\tilde{r}_{0}$ is also bounded. The same limits in $(2.2 \mathrm{~b})$ give the equation:

$$
\ddot{\theta}=-\frac{g+\ddot{z}}{L+\epsilon \tilde{r}} \sin \theta-\frac{2}{L+\epsilon \tilde{r}} \epsilon \dot{\tilde{r}} \dot{\theta}-\frac{\beta}{m} \dot{\theta}-\frac{\beta \dot{\bar{z}}}{m(L+\epsilon \tilde{r})} \sin \theta
$$



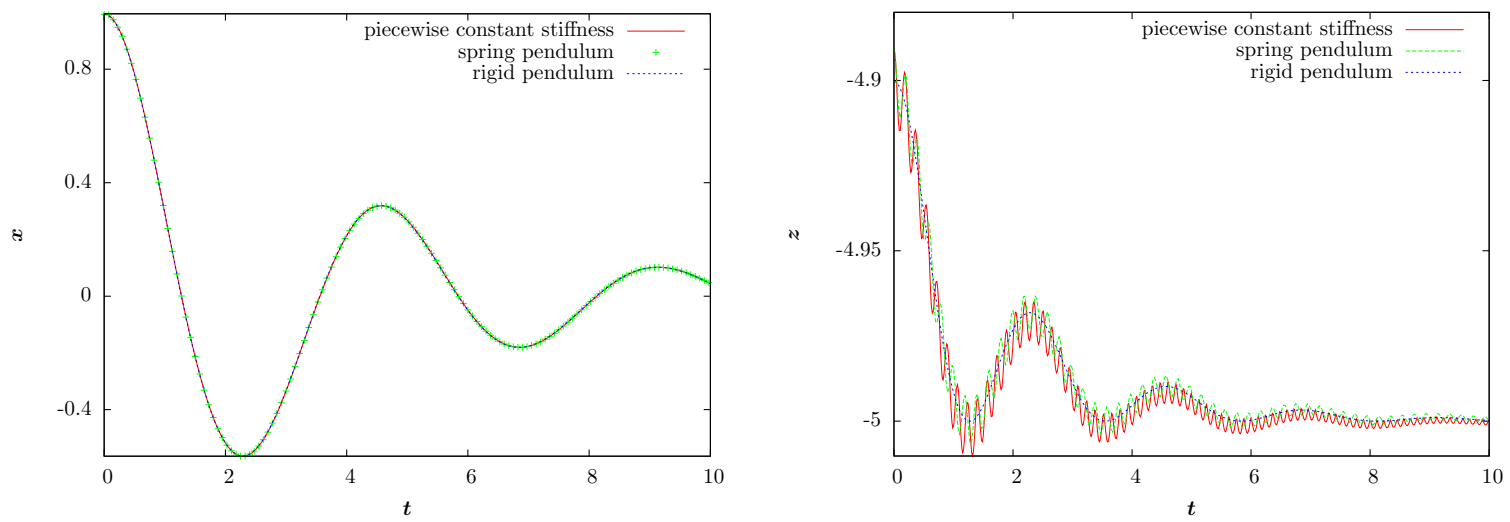

Figure 7: For small angle oscillations, this shows a good match of the time profiles for the motion of the spring pendulum with piecewise constant stiffness, the simple spring pendulum and the rigid pendulum. Parameter values used are $A=0, L=5, g=9.8, \beta=0.5$, $k_{1}=1000, k_{2}=0.01$ (for piecewise constant stiffnesses) and $k=1000$ (for the simple spring pendulum).

Taking equation (2.6) in the limit $\epsilon \rightarrow 0$, we note that the term $L+\epsilon \tilde{r}$ in the denominators approach $L$ and the second term on the right hand side is $\mathscr{O}(\epsilon)$ and thus can be ignored to leading order. Therefore we conclude that in the limit $k \rightarrow \infty$ equations (2.2) reduce to the equation for the forced damped pendulum (1.4). The spring pendulum approach for modeling the rigid pendulum is thus appropriate for large spring constants $k$.

\subsection{Spring pendulum with piecewise constant stiffness}

So far we have considered a spring pendulum with a large spring constant so that it behaves similarly to the classic pendulum resisting stretching and compression. In moving towards the string pendulum that can become slack under a vertical forcing $\bar{z}(t)$, the model of the spring pendulum needs to be adapted to model such slack states.

We thus consider the spring pendulum with piecewise constant stiffness, characterized by different spring constants $k_{1}$ and $k_{2}$ depending on whether the spring is extending or compressing relative to the fixed, original length of the string [6]. If $k_{1}$ is the constant associated with extension $r>0$, this parameter should maintain a high value to prevent the pendulum from extending beyond its length $L$. If $k_{2}$ corresponds to compression $r<0$, than this value should be relatively small to allow for some flexibility and low resistance to compression. Numerical simulation of the equations of motion suggested that $k_{1}=1000$ and $k_{2}=0.01$ are sufficiently high and respectively low values for these constants.

The equations of motion will be analogous to equations (2.2a) and (2.2b), but will include a condition that checks whether the pendulum is compressed or extended. This is controlled by the variable $r$ : 


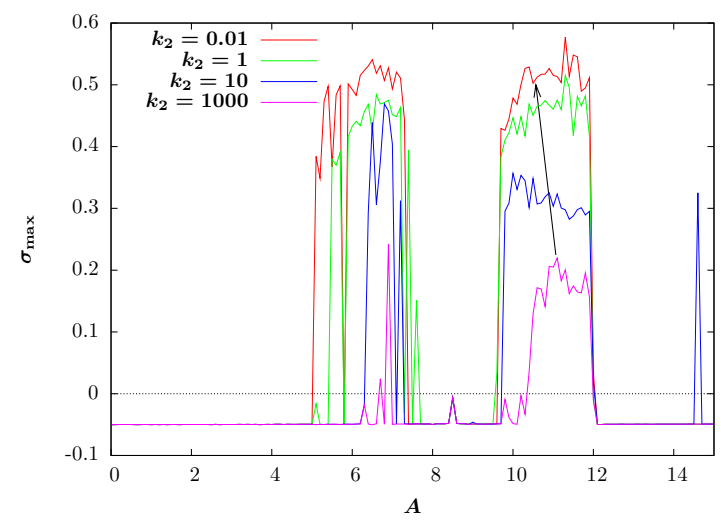

Figure 8: The dependence of the largest Lyapunov exponent for the spring pendulum with piecewise constant stiffness on amplitude $A$ ranging from 0 to 15 (using Wolf's method in [16]). Parameter values used are $\omega=0.9 \sqrt{g / L}, g=9.8$, damping $\beta=0.1, k_{1}=1000$, and $k_{2}=0.01, k_{2}=1, k_{2}=10, k_{2}=1000$ (uniform stiffness case).

$$
\begin{gathered}
\kappa(r)= \begin{cases}k_{2} & \text { if }-L<r<0, \\
k_{1} & \text { else. }\end{cases} \\
\ddot{r}=\ddot{\bar{z}} \cos \theta+(L+r) \dot{\theta}^{2}+g \cos \theta-\frac{\kappa(r) r}{m}-g-\frac{\beta}{m} \dot{r}+\frac{\beta \dot{\bar{z}}}{m} \cos \theta .
\end{gathered}
$$

The equation of motion for $\theta$ is unchanged from equation $(2.2 \mathrm{~b})$. The choice for the switching condition in $\kappa(r)$ is motivated by the extension or compression of the spring, as well as to avoid the singularities that would happen if $L+r \rightarrow 0$.

Fig. 7 provides a comparison of the spring pendulum with piecewise constant stiffness with the simple spring pendulum analyzed earlier in this section, as well as the rigid pendulum in Section 1.1. We consider the unforced case with $\bar{z}(t) \equiv 0$ and note that the motions in the $x$ direction match almost indistinguishably for small angle oscillations, while the $z$ behaviors differ. We choose to compare the $x, z$ directions as opposed to the $r, \theta$ ones because the DAE approach in section 3 also uses Cartesian coordinates. We note that the slow, damped oscillations in the $z$ direction for the spring pendulum are due to the large value of the spring constant $k$, while the faster oscillations for the spring pendulum with piecewise constant stiffness are due to the large $k_{2}$ and very small $k_{1}$, which allow the string to have a lower resistance to compression.

A Lyapunov exponent dependence on a range of amplitudes for the spring pendulum with piecewise constant stiffness is showed in Fig. 8. The dependence of the Lyapunov exponent evolution on the value of the compression constant $k_{2}$ is explored in this figure. Since the spring pendulum with piecewise constant stiffness is a model of the string pendulum, we cannot expect it to be in perfect agreement with Fig. 4, which is a formulation of the classic pendulum. We note that large values of $k_{2}\left(k_{2}=1000\right.$, and similarly $k_{2}=100$ ) lead to a Lyapunov exponent plot similar to the one in Fig. 6, with a chaotic range of amplitudes around $A=10-12$ and stable solutions for small amplitudes. The amplitude of the largest Lyapunov exponents for this chaotic range 
is also similar, with maximum value around 0.2 . This is expected because the case $k_{2}=k_{1}=1000$ corresponds to the uniform stiffness pendulum considered in Section 2. As the constant $k_{2}$ is decreased, the Lyapunov exponents for the chaotic range become larger, and an additional region of chaotic behavior is observed at amplitudes $A=5-7$. The model of the string pendulum, with $k_{2} \rightarrow 0$, has a larger range of amplitudes where solutions are expected to be chaotic.

The string pendulum can thus be approximated with the model of a spring pendulum with piecewise constant stiffness, which allows for the string of the pendulum to become slack under some exterior forcing amplitudes. An analysis of the accuracy of this approximation is provided in Section 5. A comparison with the modeling method of the string pendulum introduced in Section 4 will help identify the advantages and disadvantages of considering a spring pendulum with piecewise constant stiffness.

\section{DAE formulation of the classic pendulum}

Section 1.1 offered a way of modeling the rigid pendulum that stays taut at all times. The system described by equation (1.4) can also be modeled using a differentialalgebraic equation system (DAE), which is a system of ODE's to which we can apply the Euler-Lagrange method. The Lagrangian will include an extra term based on a geometric constraint with a Lagrange multiplier. In Section 4, the DAE system will be extended to consider the case of a flexible pendulum string.

Since we want to work in the stationary reference frame (and thus keep track of the vertical motion $z-\bar{z}$, which will prove useful when switching from taut to slack states), we will be working with $x, z$ coordinates as opposed to the polar coordinates we used so far. In our case, the geometric condition is a rheonomic constraint [4] coming from the fact that the pendulum string is inextensible and thus restricts the motion of the mass:

$$
f(\lambda) \equiv x(t, \lambda)^{2}+(z(t, \lambda)-\bar{z}(t))^{2}-L^{2},
$$

and we require that $f(\lambda)=0$. The Lagrange multiplier $\lambda(t)$ may be interpreted as being related to the tension force in the string.

The Lagrangian $\mathscr{L}=\mathscr{L}(x, z, \lambda)=\mathscr{L}(\boldsymbol{u})$ becomes

$$
\mathscr{L}=T-U=\frac{1}{2} m\left(\dot{x}^{2}+\dot{z}^{2}\right)-m g z-\lambda\left(x^{2}+(z-\bar{z})^{2}-L^{2}\right) .
$$

From the Euler-Lagrange equation (1.2) with respect to each variable in $\boldsymbol{u}=$ $(x, z, \lambda)$ and the Rayleigh dissipation function, which is again taken to be of the form $F=\frac{\beta}{2}\left(\dot{x}^{2}+\dot{z}^{2}\right)$, we can deduce

$$
\begin{aligned}
\ddot{x} & =-\frac{2 \lambda x}{m}-\beta \dot{x}, \\
\ddot{z} & =-g-\frac{2 \lambda(z-\bar{z})}{m}-\beta \dot{z} \\
0 & =x^{2}+(z-\bar{z})^{2}-L^{2} .
\end{aligned}
$$


In order to solve this system, at each time step in a numerical simulation we need to determine $\lambda$ so that (3.2c) is satisfied. We do this by evolving equations (3.2a) and (3.2b) and checking if condition (3.2c) is satisfied. If it is not, we adjust $\lambda$ and redo the evolution of $x$ and $z$. We adjust $\lambda$ using Newton's method to converge to the $\lambda(t)$ value for each time step:

$$
\lambda_{k+1}=\lambda_{k}-\frac{f\left(\lambda_{k}\right)}{f^{\prime}\left(\lambda_{k}\right)}
$$

with $f$ given by (3.1).

A method of computing the denominator in the above equation that is numerically more efficient is to differentiate the expression for $f$ in terms of $x$ and $z$ as a function of $\lambda$. Thus, starting with $f(\lambda)=x(\lambda)^{2}+(z(\lambda)-\bar{z})^{2}-L^{2}$, we obtain $f^{\prime}(\lambda)=2 x \frac{\partial x}{\partial \lambda}+$ $2(z-\bar{z}) \frac{\partial z}{\partial \lambda}$. We will call the derivatives of $x$ and $z$ with respect to $\lambda$ as $\eta$ and $\xi$, then it follows that $f^{\prime}(\lambda)=2 x \eta+2(z-\bar{z}) \xi$.

Therefore, we have two extra ODE's coming from taking derivatives with respect to $\lambda$ of the original equations $(3.2 \mathrm{a}),(3.2 \mathrm{~b})$. The system under consideration becomes:

$$
\begin{aligned}
\ddot{x} & =-\frac{2 \lambda x}{m}-\beta \dot{x}, \\
\ddot{z} & =-g-\frac{2 \lambda(z-\bar{z})}{m}-\beta \dot{z}, \\
\ddot{\eta} & =-\frac{2}{m} x-\frac{2 \lambda}{m} \eta-\beta \dot{\eta}, \\
\ddot{\xi} & =-\frac{2}{m}(z-\bar{z})-\frac{2 \lambda}{m} \xi-\beta \dot{\xi}, \\
0 & =x^{2}+(z-\bar{z})^{2}-L^{2} .
\end{aligned}
$$

The initial conditions for $x$ and $z$ are unchanged, and $\eta=\dot{\eta}=0$ and $\xi=\dot{\xi}=0$ since we will compute $\lambda$ independently on each time step.

Thus there is no ODE for the $\lambda$ variable, but the value from the algorithm above is determined indirectly from $x$ and $z$ so that they satisfy (3.4e). Then once $\lambda$ is known, equations (3.4a) and (3.4b) determine the evolution of $x(t)$ and $z(t)$.

The simulation based on the DAE system and Newton's method implies using equation (3.3) for a number of iterations for each time step. Six iterations suffice for convergence of Newton's method in this case, and the numerical scheme is first order accurate. Since the Runge-Kutta integration is called in each call of function $f$, the integration is performed several times for each time step. This is more complicated and takes more time than the simulation for the spring pendulum model, which involves using a simple Runge-Kutta integration applied to equations (2.2a) and (2.2b). However, the method presented in this section describes the string pendulum system by definition and avoids the oscillations around the trajectories obtained in the spring pendulum model.

We note that the simulation described in Section 2 matches the simulation generated using the DAE formulation well. Moreover, Fig. 9 shows an evolution of the largest Lyapunov exponent of the system for a range of $A$ 's that matches closely the one 


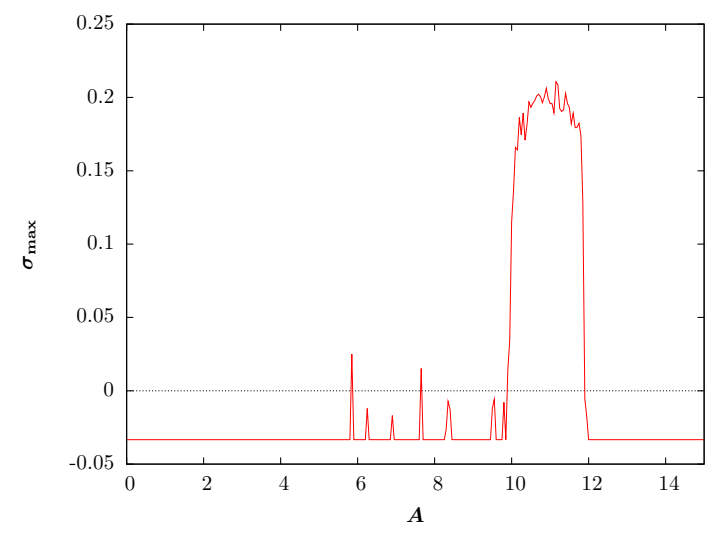

Figure 9: Parameter dependence of the largest Lyapunov exponent for the forced damped pendulum modeled using the DAE formulation on amplitude $A$ ranging from 0 to 15 . Parameter values used are $L=5, g=9.8, \omega=0.9 \sqrt{g / L}, \beta=0.1$.

for the classic and spring pendulums, see Fig. 4 and 6 . The excellent agreement with Fig. 4 was expected since both the DAE formulation and the one in Section 1.1 describe the same physical system of a classical pendulum with non-stretchable and non-compressible string. This agreement over the whole parameter range shows that the method of determining the largest Lyapunov exponent of a system described by equation (1.6) at the end of Section 4 and used for the DAE formulation matches the methods in [16] and [2] used for the classic and spring pendulum systems. While [16] and [2] use the linearized equations for the nearby trajectory $\boldsymbol{u}_{\mathbf{1}}$ and give all the Lyapunov exponents of the system, the method applied to the DAE system uses only two nearby trajectories on the full system and only determines the largest Lyapunov exponent $\sigma_{\max }$.

We will now use this DAE formulation to set up the taut-slack problem for the string pendulum in the next section.

\section{The taut-slack pendulum}

Section 3 offers a way of modeling the rigid pendulum with external forcing. In order to analyze the dynamics of the string pendulum, we need to consider the possibility of the pendulum string becoming slack, i.e. $x^{2}+(z-\bar{z})^{2}<L^{2}$. Given enough velocity, the mass can jump and follow a parabolic path until the string is extended again [5]. Thus, we need to explore how switching from a rigid rod of length $L$ to a massless string of length $L$ will change the behavior of the system. The simulation of the string pendulum will cause some challenges in detecting the switching conditions between the taut and slack cases.

The initial conditions for the forced string pendulum can be either taut or slack. If the problem's initial conditions are taut, then the DAE formulation in Section 3 holds for at least one more time step and equations (3.4) can be applied. If the initial conditions of the system are slack, i.e. $x^{2}+(z-\bar{z})^{2}<L^{2}$, we would have the equations 

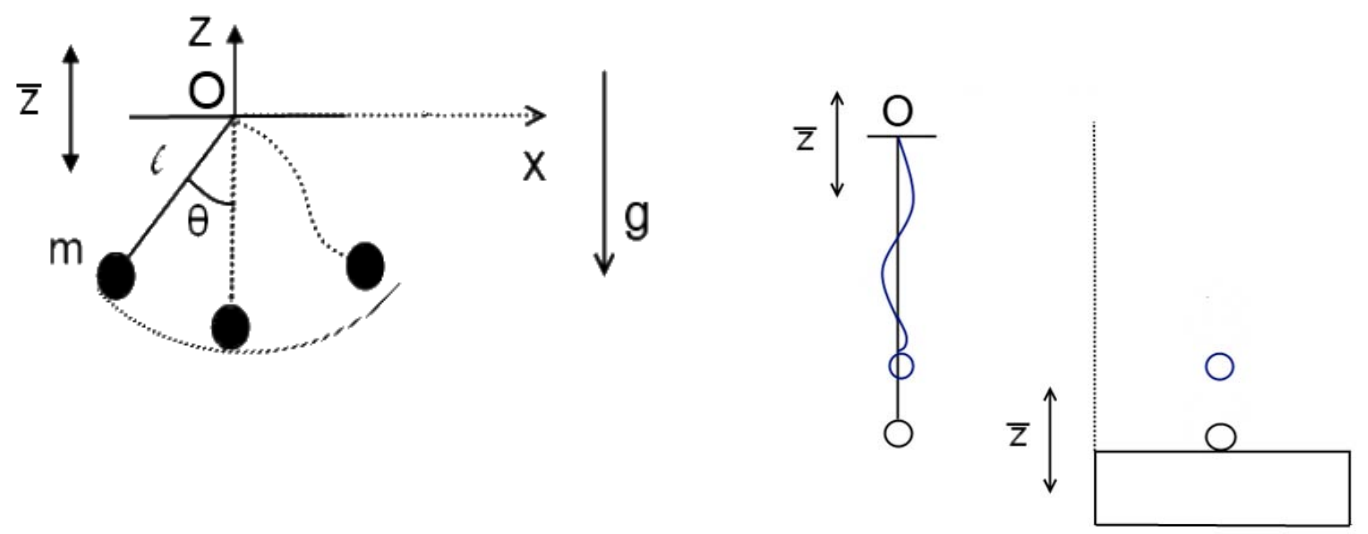

Figure 10: This figures shows (a) A schematic diagram of the string pendulum in both the taut and slack states and (b) A string pendulum under exterior forcing with no horizontal motion

of motion:

$$
\ddot{x}=-\frac{\beta}{m} \dot{x}, \quad \ddot{z}=-g-\frac{\beta}{m} \dot{z},
$$

since the mass of the pendulum would just be in free fall and thus only affected by gravity and damping due to air resistance. We note that the above equations with the change of variables $x=(L+r) \sin \theta, z=\bar{z}-(L+r) \cos \theta$ yield equations (2.2) with the exception of the spring restoring force in the term $-\frac{k r(t)}{m}-g$. This shows that the spring pendulum and taut-slack formulations are consistent. For the piecewise constant stiffness model, the $r<0$ case (slack) corresponds to the compression constant $k_{2} \rightarrow 0$, which means that the restoring force vanishes and thus matches equation (4.1) for the slack case.

In the case of starting in the taut pendulum state, but with velocities in the $x$ and $z$ directions creating a positive outward normal velocity due to a previous slack state, switching conditions need to be determined in order to prevent the pendulum string from stretching out. The equations of motion will still be given by equations (3.4) and our main focus now is on switching between these dynamical states.

\subsection{Switching conditions in absence of horizontal motion}

A simulation using equations (3.4) for the taut case and equations (4.1) for the slack case fails to take into account the fact that the pendulum string cannot stretch for a length greater than $L$. In order to solve this problem, we first reduce it to an easier one, a one-dimensional problem for a particle that can only move in the $z$ direction. There is no forcing in the $x$ direction, and if $x(0)=0$ then $x(t) \equiv 0$ for any $t$. We thus reduce the problem to the $1 \mathrm{D}$ case. The Lagrangian in this case is $\mathscr{L}=T-U=\frac{1}{2} m \dot{z}^{2}-m g z$.

To gain insight into the solution to this problem, we identify the similarity between this situation and the one of the ball positioned on a sinusoidally-vibrating table, which also exhibits a switching behavior [3], see Fig. 10b. Using the insight gained from this 
situation, the acceleration equation is

$$
\ddot{z}= \begin{cases}\ddot{z}-\beta \dot{z} & \text { if } z \leq \bar{z}-L \text { and }(\dot{z}-\dot{\bar{z}})(z-\bar{z})>0, \\ -g-\beta \dot{z} & \text { else. }\end{cases}
$$

We note that we should also take into account the fact that our Euler-Lagrange approach might be affected by the "corner conditions" imposed at times when the mass of the pendulum is going from slack to fully stretched and vice versa [10]. Based on the analogy between impacts of the ball bouncing off table and pendulum mass being constrained by a string, we write a corner condition relating the velocity immediately before and after impact (at time $t_{*}$ ). We use $c$ to denote the coefficient of restitution and thus $c=1$ for an elastic rebound (stretchy string), $0<c<1$ for a partially inelastic rebound and $c=0$ for a completely inelastic impact (unstretchable string). The corner condition is given in the moving reference frame by:

$$
\dot{z}\left(t_{*}^{+}\right)=-c \dot{z}\left(t_{*}^{-}\right),
$$

so that in the stationary reference frame we obtain $\dot{z}\left(t_{*}^{+}\right)=\dot{\bar{z}}-c \dot{z}\left(t_{*}^{-}\right)$.

\subsection{Switching conditions for general motion}

Now we are left with finding corner conditions for the case of the $2 \mathrm{D}$ pendulum. Our physical understanding of the system indicates that, in order for the no-stretch condition to be satisfied, we would need to check the velocity in the normal direction of the pendulum when it reaches a taut position. If the normal component of the velocity is greater than zero, than it needs to be zeroed in the code so that the simulation does not allow the string to go over its maximum length of $L: x^{2}+(z-\bar{z})^{2}=L^{2}$. A similar idea is introduced in [5], where the radial component of the velocity of the mass is zeroed when the string becomes taut in the case of a toy jumping pendulum.

The normal vector to the trajectory of the pendulum when it makes an angle $\theta$ with the vertical is $\boldsymbol{n}=(\cos \theta, \sin \theta)$, while the vector tangent to this trajectory is $\boldsymbol{t}=(\sin \theta,-\cos \theta)$.

We will denote by $\boldsymbol{v}^{-}$the velocity of the string before reaching the taut position and by $\boldsymbol{v}^{+}$the velocity right after this position. We will therefore have an equation of $\boldsymbol{v}^{-}$in terms of $\boldsymbol{v}_{n}^{-}$and $\boldsymbol{v}_{t}^{-}$and an equation relating $\boldsymbol{v}^{+}$to $\boldsymbol{v}_{n}^{+}$and $\boldsymbol{v}_{t}^{+}$. We will try to find the values of the latter two velocities that correspond to our physical understanding of the system as described above. We also denote the norm of these vectors by: $v_{n}^{-}=\left\|\boldsymbol{v}_{\boldsymbol{n}}^{-}\right\|$

and $v_{t}^{-}=\left\|\boldsymbol{v}_{\boldsymbol{t}}^{-}\right\|$. We will consider the system in the moving reference frame in the following derivation.

We start with $\boldsymbol{v}^{-}=v_{n}^{-} \boldsymbol{n}+v_{t}^{-} \boldsymbol{t}=(\dot{x}, \dot{z})$. We can thus find $\boldsymbol{v}_{n}^{-}$and $\boldsymbol{v}_{t}^{-}$by finding the projection of $\boldsymbol{v}^{-}$on the normal and tangential directions:

$$
\boldsymbol{v}_{n}^{-}=(\dot{x} \cos \theta+\dot{z} \sin \theta)(\cos \theta, \sin \theta), \quad \boldsymbol{v}_{t}^{-}=(\dot{x} \sin \theta-\dot{z} \cos \theta)(\sin \theta,-\cos \theta) .
$$

Similarly, one can obtain equations for the states following the taut position: 

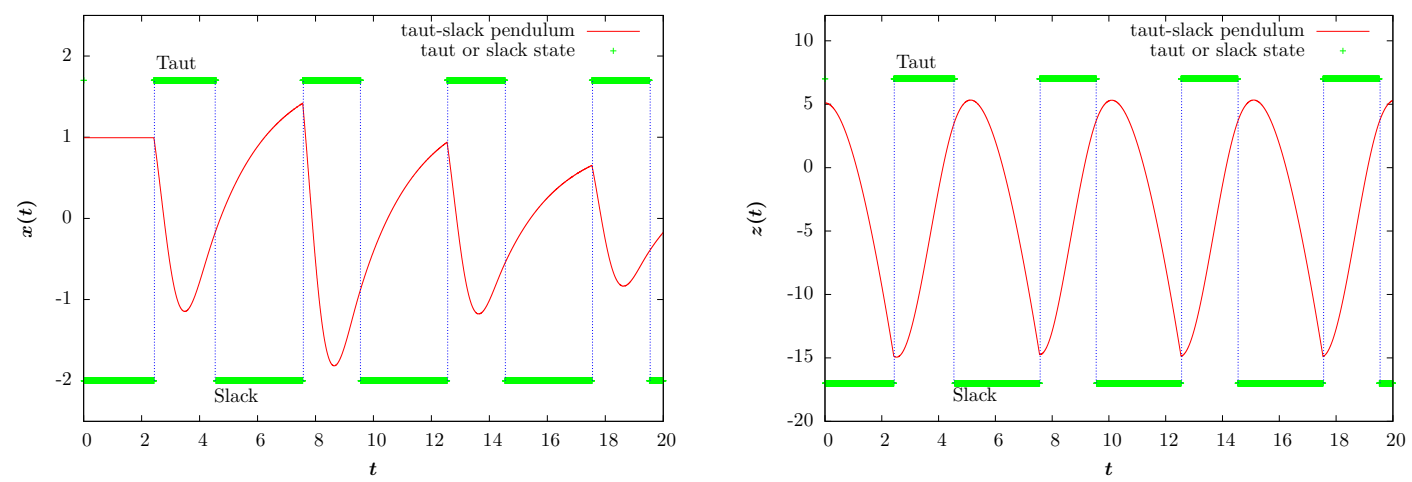

Figure 11: The time profiles in the $x$ (left) and $z$ (right) coordinates for the forced string pendulum modeled using the taut-slack formulation. Parameter values used are $A=10$, $\omega=0.9 \sqrt{g / L}, L=5, g=9.8$, damping $\beta=0.5$.

$$
\boldsymbol{v}_{n}^{+}=\left(\dot{x}^{+} \cos \theta+\dot{z}^{+} \sin \theta\right)(\cos \theta, \sin \theta), \quad \boldsymbol{v}_{t}^{+}=\left(\dot{x}^{+} \sin \theta-\dot{z}^{+} \cos \theta\right)(\sin \theta,-\cos \theta) \text {. }
$$

The relation between the two states is summarized as

$$
\boldsymbol{v}_{n}^{+}=-c \boldsymbol{v}_{n}^{-}, \quad \boldsymbol{v}_{t}^{+}=\boldsymbol{v}_{t}^{-},
$$

for $c$ representing the coefficient of restitution.

We solve the system of equations (4.4) and obtain:

$$
\dot{x}^{+}=\dot{x}\left(\sin ^{2} \theta-c \cos ^{2} \theta\right)+\dot{z}(-\sin \theta \cos \theta-c \sin \theta \cos \theta),
$$

and

$$
\dot{z}^{+}=\dot{x}(-c \sin \theta \cos \theta-\sin \theta \cos \theta)+\dot{z}\left(\cos ^{2} \theta-c \sin ^{2} \theta\right) .
$$

Using that $\sin \theta=\frac{z-\bar{z}}{L}$ and $\cos \theta=\frac{x}{L}$, as well as the assumption of inelastic collision $c=0$, we can find the values for the velocities in the $x$ and $z$ directions:

$$
\dot{x}^{+}=\dot{x} \frac{(z-\bar{z})^{2}}{L^{2}}-(\dot{z}-\dot{\bar{z}}) \frac{x(z-\bar{z})}{L^{2}}, \quad \dot{z}^{+}=\dot{\bar{z}}-\dot{x} \frac{x(z-\bar{z})}{L^{2}}+(\dot{z}-\dot{\bar{z}}) \frac{x^{2}}{L^{2}} .
$$

We can therefore summarize the switching between the taut and slack states of the string pendulum as follows:

1. If the string is taut $\left(x^{2}+(z-\bar{z})^{2}=L^{2}\right)$ and stretching out $(x \dot{x}+(z-$ $\bar{z})(\dot{z}-\dot{\bar{z}})>0)$, then:

(a) If the string was previously slack, apply equations (4.5) to determine the updated velocities and then use equations of motion (3.4).

(b) If the string was previously taut, use equations of motion (3.4).

2. If the string is slack, then use equations of motion for free fall (4.1). 

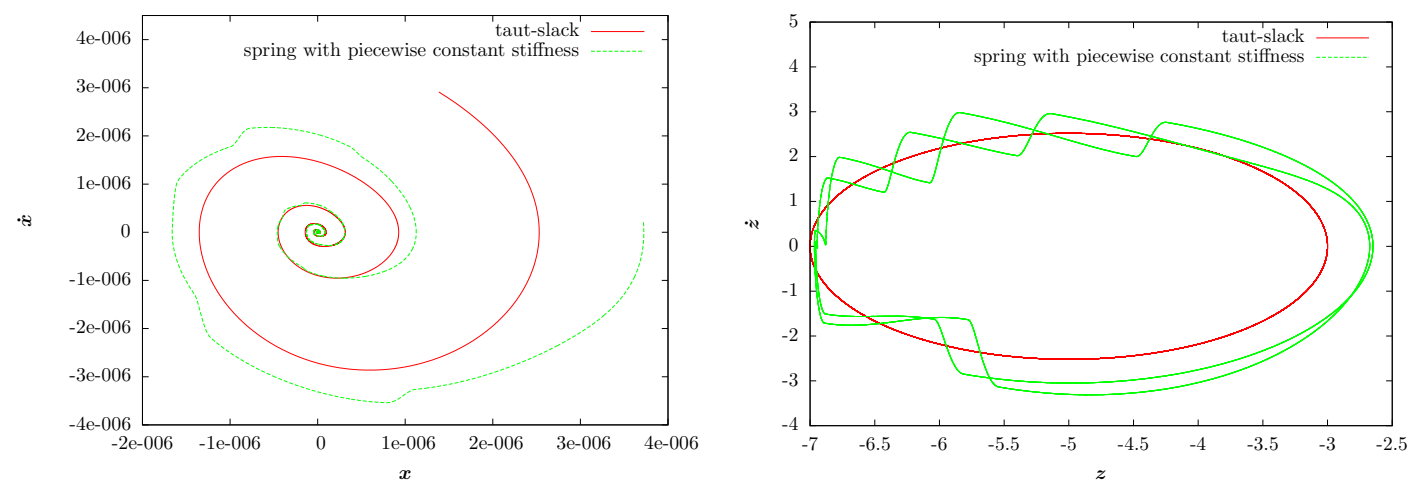

Figure 12: This shows agreement in phase planes in the $x$ (left) and $z$ (right) coordinates for the forced string pendulum modeled using the taut-slack formulation and the spring pendulum with piecewise constant stiffness. Parameter values used are $A=2, \omega=0.9 \sqrt{g / L}$, $L=5, g=9.8$, damping $\beta=0.5$.

This algorithm will be used in the next section in order to perform numerical simulations of the taut-slack system and compare its behavior with that of the spring pendulum with piecewise constant stiffness formulation.

\section{Comparison of the DAE and spring models of the taut-slack pendulum}

In Section 2.1 we identified a method of modeling the string pendulum that can become slack by considering a spring pendulum with piecewise constant stiffness (equations $(2.2 \mathrm{~b})$ and $(2.7))$. This method will be compared against the model of a string pendulum that switches between the taut and slack positions using the conditions identified in Section 4.2.

First, we note the $x$ and $z$ trajectories of motion for the taut-slack formulation in Fig. 11, which also indicates the times when the string pendulum is taut (green line above trajectory) and slack (green line below trajectory). For an amplitude of $A=10$, the string pendulum thus goes through alternating taut and slack states.

In order to compare these two models of the string pendulum, we first consider the case of no forcing $(A=0)$. The two approximations match very well for the unforced case, as it can be checked with a simple simulation of the $x$ and $z$ trajectories.

The dynamics generated by the taut-slack formulation and the spring pendulum method can be further analyzed through phase planes at different amplitudes. In Fig. 12 we consider amplitude $A=2$ in order to show the dynamics of the string pendulum for small amplitude. The $x$ vs $\dot{x}$ plot to the left is an inward spiral for both the tautslack and the spring pendulum with piecewise constant stiffness formulations. The small values obtained after ignoring the transients mean that the oscillations in the $x$ direction keep getting smaller, so that the solutions approach steady solutions in the moving reference frame (with $x(t) \equiv 0$ and $z(t) \equiv \bar{z}(t)$ ). The $z$ vs $\dot{z}$ phase plane to the 

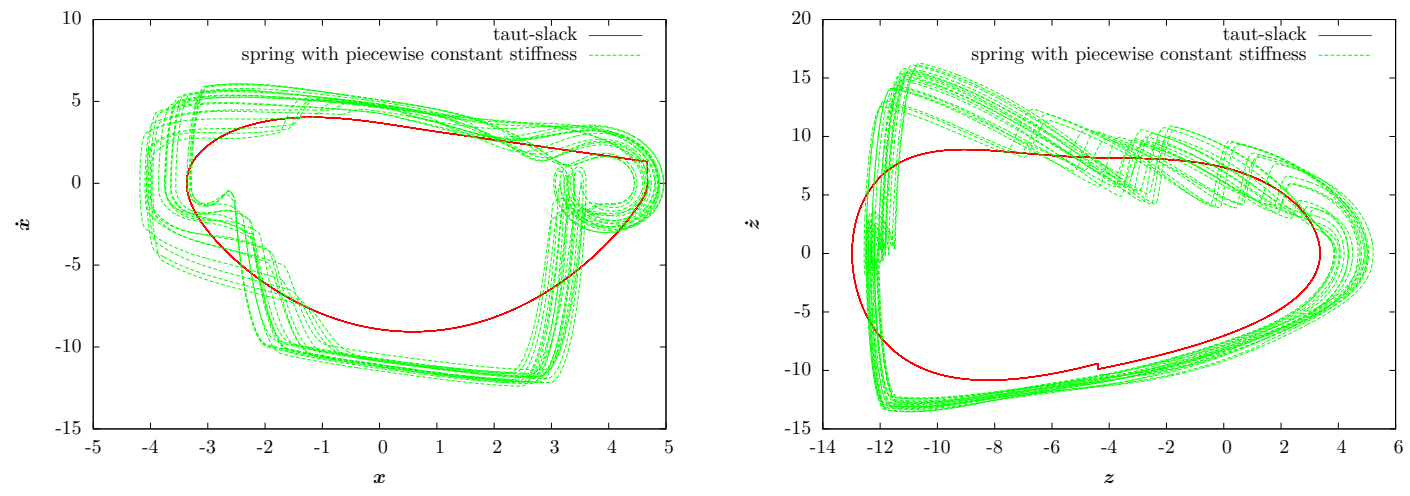

Figure 13: This shows some disagreement in phase planes in the $x$ (left) and $z$ (right) coordinates for the forced string pendulum modeled using the taut-slack formulation and the spring pendulum with piecewise constant stiffness. Parameter values used are $A=8$, $\omega=0.9 \sqrt{g / L}, L=5, g=9.8$, damping $\beta=0.5$.

right is a periodic cycle, with the plane for the spring pendulum generally following the one for the taut-slack case in a less smooth way. This is an example of a stable state in which the string pendulum is going mostly up and down with the forcing, with little motion in the $x$ direction after early transients have passed. The two models agree in this case and have the same stable behavior.

Fig. 13 shows the phase planes in the $x$ and $z$ components for the models under comparison for $A=8$. While the plot to the right shows a similar stable periodic solution for both the taut-slack and the spring pendulum approaches, the plot to the left displays an interesting difference in the stability behavior of the two models for the same amplitude $A=8$. The phase plane of $x$ vs $\dot{x}$ for the taut-slack pendulum is a periodic cycle, but the corresponding phase plane for the spring pendulum with piecewise constant stiffness shows a chaotic solution, despite the fact that it generally follows the stable solution of the taut-slack formulation. This points to the differences between the two formulations, which can give different solutions from the point of view of their stability.

We can observe the effects of further increasing the forcing amplitude $A$ in Fig. 14. The plot to the right is the phase plane of $z$ vs $\dot{z}$ for the largest amplitude in the considered range, $A=15$. We note that both the taut-slack formulation and the spring pendulum with piecewise constant stiffness have a stable periodic solution, despite the fact that the $z$ component of the spring pendulum model covers a slightly higher range of values and reaches to the top of the pendulum. The $z$ vs $\dot{z}$ plot shows that both solutions go through both taut and slack states, with vertical lines representing a switch between states. Similarly, the $x$ component shows an agreement in the general form of the phase planes of the two formulations, with periodic solutions that form several loops around the origin. Both the time profile and the velocities in the $x$ direction seem to be covering a higher range of values, showing that the two models are not in perfect agreement for higher amplitudes of the forcing.

We can note that the two methods used in modeling the string pendulum are overall 

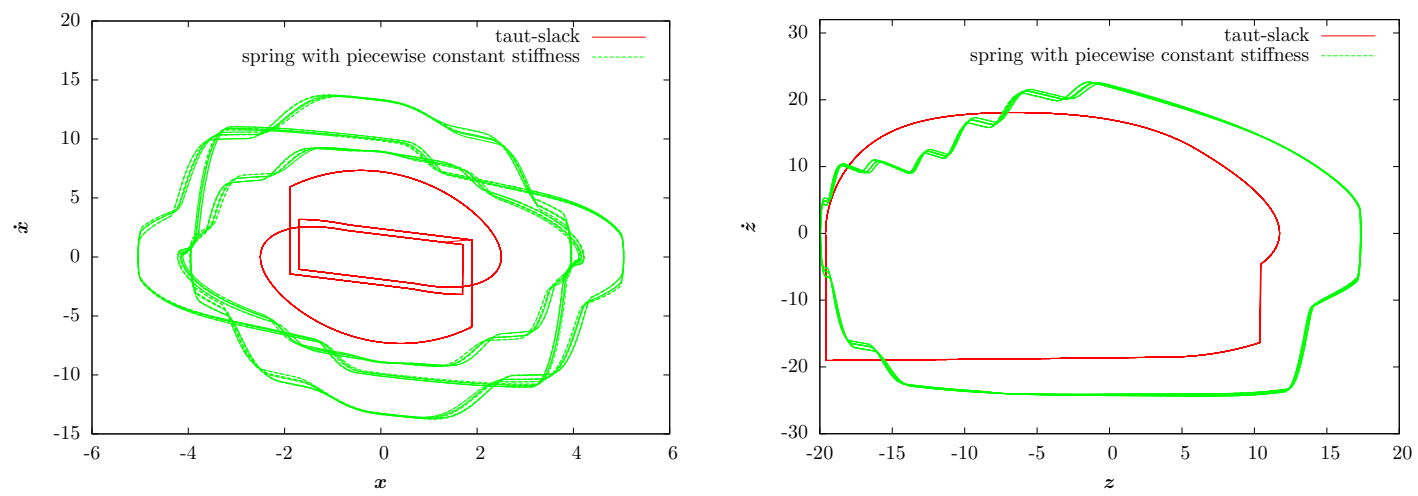

Figure 14: This shows agreement in phase planes in the $x$ (left) and $z$ (right) coordinates for the forced string pendulum modeled using the taut-slack formulation and the spring pendulum with piecewise constant stiffness. Parameter values used are $A=15, \omega=0.9 \sqrt{g / L}$, $L=5, g=9.8$, damping $\beta=0.5$.

similar in the stability of the solutions, despite differing for certain amplitudes $(A=8$ in Fig. 13). Fig. 8 in Section 2 shows a dependence of the largest Lyapunov exponent for the spring pendulum with piecewise constant stiffness on a range of amplitudes. Such a plot is difficult to produce for the case of the DAE modeling method, because of the resetting of velocities at times when the string pendulum might go from a slack to a taut case. The phase planes in this section were used instead of a Lyapunov exponent analysis in order to compare the stability of the behaviors generated by the two models. We note that the spring pendulum with piecewise constant stiffness has the advantage of providing a good, simple approximation of the forced string pendulum, while the DAE model is a formulation of the string pendulum that requires a more complex algorithm and switching between two sets of equations of motion.

\section{Conclusions and further work}

We have modeled the classic pendulum using both a spring pendulum and a differential algebraic system (DAE) approach. The string pendulum, which is not as well understood as the classic pendulum, was approximated using a spring pendulum with piecewise constant stiffness and a DAE system with switching between the taut and slack cases. These modeling methods proved to match very well, as proven by the time profiles and phase planes of the trajectories. Moreover, the dependence of the largest Lyapunov exponent of the system on parameter $A$ denoting amplitude of the forcing shows that the stability behavior and dynamics of the approximations are also similar.

More work can be done in determining the Lyapunov exponents of the DAE formulation for the string pendulum. This would allow a complete comparison of this method with the spring with piecewise constant stiffness.

A more thorough study of the formulations' dependence on parameters would include holding forcing amplitude $A$ fixed and changing the forcing frequency $\omega$, which could be used to study resonant responses. We note that the values of the length $L$ and 
amplitude $A$ considered here may be relevant for applications such as moored boats, but the dynamics for other motivations should be investigated for smaller values of these parameters. Another possibility for future work is looking for multiple stable states by starting from different initial conditions at the same system parameters. Alternatively, the system's behavior could be numerically simulated using continuation for a finite range of amplitudes $A$; decreasing $A$ back to its starting value would allow to check if hysteresis occurs, in the case where multiple solutions are obtained.

Leading to an analysis of Newton's cradle, a future step in this research will be considering a string pendulum suspended by two cables attached to a frame which is given an exterior forcing. This will be the case of having one ball in the Newton's cradle setup in Fig. 1c.

\section{Acknowledgments}

The paper is a result of the work done for the Duke University PRUV Fellow program during summer 2010 and the Math 192 independent study taken during Spring 2011, under the guidance of Professor Thomas Witelski, and NSF CMMI grant \# 0927186: New challenges in Non-smooth Dynamical systems.

\section{References}

[1] M. V. Bartuccelli, G. Gentile, and K. V. Georgiou, On the dynamics of a vertically driven damped planar pendulum, Proceedings: Mathematical, Physical and Engineering Sciences, 457 (2001), pp. 3007-3022.

[2] F. Christiansen And H. H. Rugh, Computing Lyapunov spectra with continuous Gram-Schmidt orthonormalization, Nonlinearity, 16 (1997), pp. 1063-1072.

[3] T. Gilet, N. Vandewalle, and S. Dorbolo, Completely inelastic ball, Physical Review E, 79 (2009), pp. 055201 1-4.

[4] H. Goldstein, Classical Mechanics, Addison-Wesley Pub. Co., 1980.

[5] A. Goriely, P. Boulanger, and J. Leroy, Toy models: The jumping pendulum, American Journal of Physics, 74 (2006), pp. 784-788.

[6] L. D. Humphreys And P. J. McKenna, When a Mechanical Model goes Nonlinear: Unexpected Responses to Low-Periodic Shaking, the American Mathematical Monthly, 112 (2005), pp. 861-875.

[7] J. B. Keller, Ponytail motion, Society for Industrial and Applied Mathematics, 70 (2010), pp. 2667-2672.

[8] J. B. Marion and S. T. Thornton, Classical dynamics of particles and systems, Saunders College Publishing, Harcourt Brace College Publishers, 4th ed., 1995. 
[9] D. Morin, Introduction to Classical Mechanics, Cambridge University Press, 2007.

[10] E. R. Pinch, Optimal Control and the Calculus of Variations, Oxford University Press, 1993.

[11] K. Popp, Non-smooth mechanical systems, Journal of Applied Mathematics and Mechanics, 64 (2000), pp. 765-772.

[12] W. H. PREss, Numerical recipes in C: the art of scientific computing, Cambridge University Press, 1997.

[13] C. Sangwin, Modelling the journey from elementary word problems to mathematical research, Notices of the AMS, 58 (2011), pp. 1436-1445.

[14] S. H. Strogatz, Nonlinear dynamics and chaos : with applications to physics, biology, chemistry, and engineering, Perseus Pub., 1994.

[15] L. N. Virgin, J. Nichols, W. Simmons, and R. Plaut, On the response of a shaken cable-suspended mass, ASME Design Engineering Technical Conference, (2001).

[16] A. Wolf, J. B. Swift, H. L. Swinney, and J. A. Vastano, Determining Lyapunov exponents from a time series, Physica D, 0545 (1985), pp. 285-317. 